\title{
Rates and risks for co-morbid depression in patients with Type 2 diabetes mellitus: results from a community-based study
}

\author{
F. Pouwer, A. T. F. Beekman, G. Nijpels, J. M. Dekker, F. J. Snoek, P. J. Kostense, R. J. Heine, D. J. H. Deeg \\ Diabetes Research Group, Department of Medical Psychology, Institute for Research in Extramural Medicine (EMGO), \\ VU University Medical Center, Amsterdam, The Netherlands
}

\begin{abstract}
Aims/hypothesis. There is accumulating evidence that depression is common in people with Type 2 diabetes. However, most prevalence-studies are uncontrolled and could also be inaccurate from selection-bias, as they are conducted in specialized treatment settings. We studied the prevalence and risk factors of comorbid depression in a community-based sample of older adults, comparing Type 2 diabetic patients with healthy control subjects.

Methods. A large $(n=3107)$ community-based study in Dutch adults (55-85 years of age) was conducted. Pervasive depression was defined as a CES-D score greater than 15. Diagnosis of Type 2 diabetes was obtained from self-reports and data from general practitioners.
\end{abstract}

Results. A number of 216 patients (7\%) were identified as having Type 2 diabetes. The prevalence of pervasive depression was increased in people with Type 2 diabetes and co-morbid chronic disease (20\%) but not in patients with Type 2 diabetes only (8\%), compared with the healthy control subjects $(9 \%)$. Regression analyses in diabetic patients yielded that being single, being female, having functional limitations, receiving instrumental support and having an external locus of control were associated with higher levels of depression.

Conclusions/interpretation. The Results suggest that the prevalence of pervasive depression is increased in patients with Type 2 diabetes and co-morbid disease(s), but not in patients with Type 2 diabetes only. Functional limitations that often accompany co-morbid chronic disease could play an essential role in the development of depression in Type 2 diabetes. These findings can enable clinicians and researchers to identify high-risk groups and set up prevention and treatment programs. [Diabetologia (2003) 46:892-898]

Keywords Type 2 diabetes mellitus, depression, prevalence, epidemiology.

diabetes was $20.5 \%$, versus $11.4 \%$ in non-diabetic subjects [1]. It was also concluded that depression does not only have a negative effect on the quality of life of people with diabetes [2] but also on their glycaemic control $[3,4,5]$, and it is associated with increased health-care use and health-care costs [6]. However, many studies aiming to determine the prevalence of depression in Type 2 diabetes are not accurate because of selection bias as they have been conducted in specialised hospitals that treat patients with problematic diabetes. Moreover, potential confounders such as sex, socio-economic status, obesity, concomitant medical illness or complications of diabetes are often not addressed. The authors therefore concluded that additional analyses are required to determine 
more definitively whether the prevalence of major depression and depression symptoms are increased in the general population of people with diabetes [1]. They also suggested that these studies should carefully measure and report potential moderators so that both adjusted and unadjusted depression prevalence can be calculated.

Knowledge about the risk factors for developing depression in Type 2 diabetic patients might also be valuable by enabling clinicians and researchers to identify high-risk groups and to set up prevention and treatment programs. Several explanations have been offered to account for the increased prevalence of depression in Type 2 diabetes. In two recent reviews, it was concluded that a large number of studies suggest that depressed mood is positively associated with the presence of diabetic complications [7, 8]. Other studies suggest that an increased level of depression is associated with demographic variables such as low education, female sex, or being unmarried $[9,10,11]$. Furthermore, general or diabetes-specific social support was found to be associated with fewer depressive symptoms or greater emotional well-being $[12,13]$. It was concluded that population-based studies are needed which combine a number of risk factors for depression, as illness intrusiveness and social support can mediate the association between disease variables and depression [7].

Therefore, the objectives of our study were to investigate the prevalence of pervasive depression in a community-based sample of older people with Type 2 diabetes, and to report both unadjusted and adjusted odds ratios for depression. Moreover, we also set out to examine the associations between depressive symptoms, co-morbid chronic diseases, functional disability, and psychosocial factors.

\section{Subjects and methods}

Sampling and response. Data were collected within the framework of the Longitudinal Aging Study Amsterdam (LASA), a study among men and women between the ages of 55 to 85 years. Data collection procedures and response have been described in detail previously $[14,15]$. In short, a random sample of community-dwelling older persons, stratified by age and sex, was drawn from the population registers of eleven municipalities in three regions of the Netherlands. The cohort was originally recruited for the study "Living Arrangements and Social Networks of Older Adults" ( $n=3805$, response-rate $62.3 \%$ ). Non-response was associated with age, sex and urbanity. Older subjects, women and those living in more urbanized areas were less likely to respond. After 10 months, between September 1992 and September 1993, participants were approached to participate in the baseline LASA interview. In total, $3107(81.7 \%)$ gave their informed consent and took part; $126(3.3 \%)$ had died; $44(1.2 \%)$ could not be contacted; 134 $(3.5 \%)$ were too ill or impaired to be interviewed; and 394 $(10.4 \%)$ refused to participate due to a lack of interest. Due to non-response on items of the depression questionnaire, a further 57 subjects were lost, leaving a study sample of 3050 for analyses pertaining to depression.
A power analysis was described in the application for a project grant of the present study. This analysis showed that at least 160 diabetic patients were needed to detect a statistical significant odds ratio of 1.5 with an alpha of 0.05 and a power of $90 \%$.

Measurements. All interviews were conducted in the homes of the participants by specially trained and intensively supervised interviewers. With permission of the participant, interviews were tape-recorded to control the quality of the data. During the interview, the following topics were assessed: diabetes (yes/no), age at onset of diabetes, type of treatment for diabetes (insulin, tablets), complications such as: cardiovascular disease (yes/no), stroke (yes/no), peripheral arteriosclerosis (yes/no) and concomitant other chronic illness. People with self-reported diabetes, who did not use insulin, or who had an onset of diabetes at an age older than 40 years were considered as having Type 2 diabetes. Functional limitations were measured with a scale previously validated in the Netherlands [16]. Symptoms of depression during the previous week were measured using the CES-D, the 20-item Center for Epidemiologic Studies Depression Scale [17]. The Dutch translation of this instrument was shown to have good psychometric properties and satisfactory criterion validity [18]. The overlap between depression and symptoms of physical illness appeared to be minimal in several studies. The CES-D total-score can range from 0 to 60 . The generally used cutoff score of 16 or more was used to identify respondents with levels of depression that were clinically significant. This level of depressive symptomatology has been referred to as pervasive depression $[17,18]$. A scale developed by Pearlin and Schooler was included to measure internal locus of control (mastery), which reflects the extent to which the respondent feels that he controls the course of his own life [19]. The amount of both instrumental and emotional social support received were also assessed [20]. Potentially confounding variables that were assessed included sex, age, level of education, partner status, smoking status, and body mass index $\left(\mathrm{kg} / \mathrm{m}^{2}\right)$.

Statistical analyses. Firstly, demographic, clinical and depression data of three groups were compared, using the Chi-square test (for dichotomous variables) and analyses of variance (continuous data): (i) subjects with Type 2 diabetes only (ii) subjects with Type 2 diabetes and co-morbid disease and (iii) subjects without a chronic disease. A $p$ value lower than 0.05 was regarded as significant. Next, logistic regression analyses were carried out to contrast the data on depression of the three groups. In analyses pertaining to pervasive depression, odds ratios with $95 \%$ confidence intervals were calculated and were adjusted for potential confounders. Stepwise linear regression analyses in patients with Type 2 diabetes were used to study whether depression could be predicted by means of the following sets of independent variables: (i) age, being single, having low education, female sex: (ii) eye problems, cardiovascular disease(s), other chronic disease(s): (iii) functional limitations, (iv) emotional/social support, instrumental social support and internal locus of control (mastery).

\section{Results}

Sample. The total study sample comprised of 3107 subjects, of whom 1578 were women (52\%) and 1920 $(62 \%)$ were married or had a partner. A number of 963 subjects were between 55 and 64 years of age $(31 \%)$, 951 were 65 to 74 years old $(31 \%)$ and 1136 were be- 
Table 1. Baseline characteristics in three different subgroups: no chronic disease, Type 2 diabetes only and Type 2 diabetes with co-morbid disease(s)

\begin{tabular}{|c|c|c|c|}
\hline & No chronic disease & Type 2 diabetes only & Type 2 diabetes with co-morbidity \\
\hline$n$ & 1197 & 51 & 165 \\
\hline Age (years) & $68 \pm 9$ & $72 \pm 8$ & $76 \pm 7 d$ \\
\hline Male sex & $52 \%(616 / 1184)$ & $53 \%(27 / 51)$ & $42 \%(69 / 162)$ \\
\hline Married/partner & $67 \%(790 / 1184)$ & $65 \%(33 / 51)$ & $57 \%(92 / 162)^{\mathrm{b}}$ \\
\hline \multicolumn{4}{|l|}{ Clinical values } \\
\hline $\mathrm{BMI}\left(\mathrm{kg} / \mathrm{m}^{2}\right)$ & $26 \pm 4$ & $27 \pm 3$ & $29 \pm 4^{d}$ \\
\hline Smoking & $29 \%(304 / 1042)$ & $23 \%(11 / 48)$ & $16 \%(21 / 128)^{b}$ \\
\hline \multicolumn{4}{|l|}{ Depression } \\
\hline Mean CES-D (depression) & $6.0 \pm 6.8$ & $6.9 \pm 7.7$ & $8.0 \pm 7.1^{\mathrm{d}}$ \\
\hline Stroke & - & - & $19 \%(31 / 165)$ \\
\hline Osteo-/Rheumatoid arthritis & - & - & $56 \%(92 / 165)$ \\
\hline Cancer & - & - & $19 \%(32 / 165)$ \\
\hline CNSLD $^{a}$ & - & - & $24 \%(40 / 165)$ \\
\hline
\end{tabular}

${ }^{\mathrm{a}}$ CNSLD, asthma, chronic bronchitis, emphysema; ${ }^{\mathrm{b}} p<0.05 ;{ }^{\mathrm{c}} p<0.01,{ }^{\mathrm{d}} p<0.001$

tween 75 and 85 years old (37\%). The even representation of men and women and the high proportion of more elderly subjects and of subjects without a partner or with chronic diseases reflect the stratified sampling procedure. Of all subjects, 1197 (39\%) reported having no chronic disease. This group of healthy subjects was used as the control group in calculating the odds for depression in Type 2 diabetes. Subjects with a chronic illness other than Type 2 diabetes were excluded from the further analyses in this study.

Type 2 diabetes mellitus. The prevalence of diagnosed diabetes (both types) was $7.8 \%$ (243 of 3107 subjects). A number of 216 subjects reported an age at onset of diabetes at 40 years or older, or reported to have diabetes but did not use insulin. These patients were therefore regarded as having Type 2 diabetes mellitus. This number of diabetic patients provided sufficient statistical power to meet the objectives of the study. The prevalence of Type 2 diabetes was $7.0 \%$ in this community-based sample of older adults. Of the patients with Type 2 diabetes, 30\% (64/216) reported to use insulin injections, 56\% (122/216) used oral drugs and $14 \%(30 / 216)$ used a diet as the main treatment for their Type 2 diabetes.

Characteristics of patients with Type 2 diabetes and healthy control subjects. Subjects without a chronic disease $(n=1197)$ were compared with patients with Type 2 diabetes only $(n=51)$ or Type 2 diabetes and comorbid chronic disease(s) $(n=165)$, using Chi-square tests (for dichotomous variables) and analyses of vari- ance (continuous data, Table 1). Several demographic, clinical and depression data of respondents with Type 2 diabetes and co-morbid chronic disease differed from those with Type 2 diabetes only, or without any chronic disease. Patients with Type 2 diabetes mellitus and co-morbid disease were considerably older, were more often single, and had lower education, a higher BMI and had a higher mean depression score. In the group of patients with Type 2 diabetes and co-morbid disease, 33 subjects ( $15 \%$ of all subjects) had only cardiovascular complications (cardiac disease, peripheral arteriosclerosis and/or stroke), 60 subjects (28\%) had only co-morbid disease that is not directly related to diabetes (CNSLD, cancer and/or osteo-rheumatoid arthritis) and 72 subjects (33\%) had both cardiovascular complications and co-morbidity not related to diabetes. Thus, in total 105 of 216 subjects (49\%) had cardiovascular complications.

Prevalence of pervasive depression. The prevalence of pervasive depression of all subjects with Type 2 diabetes was $16.9 \%$ (36 of 213 subjects). The prevalence of pervasive depression in subjects with Type 2 diabetes (no other chronic disease) was $7.8 \%$, which was not significantly different from healthy subjects (8.9\%, Table 2$)$. In the logistic regression analysis, the odds for depression were not significantly different for patients with Type 2 diabetes only and the healthy control subjects.

We found a higher prevalence of pervasive depression $(19.8 \%)$ in the group of patients with Type 2 diabetes and co-morbid chronic disease, compared with 
Table 2. Rates of pervasive depression (CES-D>15) in subjects with Type 2 diabetes only, Type 2 diabetes with co-morbid disease(s), compared with healthy subjects

\begin{tabular}{|c|c|c|c|c|}
\hline & \multirow[t]{2}{*}{$n$} & \multicolumn{3}{|c|}{ Pervasive depression } \\
\hline & & $n(\%)$ & OR $(95 \% \mathrm{CI})$ & adjusted OR (95\% CI) \\
\hline Type 2 diabetes without co-morbid disease & 51 & $7.8 \%(4 / 51)$ & $0.88(0.3-2.5)$ & $0.94(0.3-2.7)$ \\
\hline No chronic disease & 1184 & $8.9 \%(90 / 1026)$ & $1.0(-)$ & $1.0(-)$ \\
\hline Type 2 diabetes with co-morbid disease(s) & 162 & $19.8 \%(32 / 162)^{\mathrm{a}}$ & $2.89(1.0-8.6)$ & $2.52(0.8-8.5)$ \\
\hline
\end{tabular}

${ }^{a} p<0.001$ adjusted for age, sex, marital status, education, BMI and smoking

those without a chronic disease and those with Type 2 diabetes only (Table 2). Likewise, logistic regression analysis yielded a higher odds ratio of 2.53 for pervasive depression for subjects with Type 2 diabetes (with co-morbid disease) compared to subjects with no chronic disease, which was 2.0 after adjustment for the potential confounders (Table 2). The odds for pervasive depression were $2.89(1.0-8.6,95 \%$ CI) and 2.52 after adjustment for confounders (not statistically significant) for Type 2 diabetes and co-morbid disease(s), compared with Type 2 diabetes only.

The prevalence of pervasive depression was $24 \%$ in the group of subjects with merely cardiovascular comorbidity, $21 \%$ in those with non-diabetes related comorbidity and $16 \%$ in the group of subjects who had both cardiovascular and non-diabetes-related co-morbidity. In the group of patients with Type 2 diabetes and cardiovascular co-morbidity, the prevalence of pervasive depression was $30 \%(9 / 30)$ in patients who had a stroke, $19 \%(12 / 63)$ in subjects with cardiac disease and $16 \%(7 / 44)$ in subjects with peripheral arteriosclerosis.

Risk factors for depression in Type 2 diabetes. Stepwise multiple linear regression analyses were carried out in subjects with Type 2 diabetes mellitus (Table 3). In the first step, female sex was associated with increased severity of depression symptoms. In the next step, it appeared that none of the individual clinical co-morbidity characteristics (eye problems, cardiovascular diseases or other chronic diseases) was associated with depression. In the third step, having functional limitations was positively associated with depression and added another $3.8 \%$ to the explained variance, while in the final step having functional limitations, receiving instrumental social support and a low sense of mastery were associated with more depression symptoms, adding almost $9 \%$ of the explained variance.

The finding that the clinical characteristics such as cardiovascular disease were not associated with depression was unexpected. To explore this result in the group of subjects with Type 2 diabetes, firstly, we conducted an additional stepwise regression analysis that included "number of chronic diseases" (sum of the chronic diseases described in Table 1) in the second step. In this analysis, the "total number of chronic diseases" was associated with depression symptomatology in the final model, with a Beta of 0.17 , $p=0.007$.

Secondly, we compared the distribution of co-morbidity across men and women with Type 2 diabetes and co-morbid disease(s). It appeared that men had cardiovascular disease more often than women $(77 \%$ vs $53 \%, p=0.001)$ and less often cancer $(10 \%$ versus $25 \%, p=0.01)$ or osteo/rheumatic arthritis (45\% vs $65 \%, p=0.01)$. Next, we conducted other additional regression analyses, for men and women separately. These analyses were similar to those described in Table 3 (except for the variable of sex, which was excluded). In the group of men with Type 2 diabetes, the results regarding the fourth model showed that being single (Beta of $0.19, p=0.05$ ), having cardiovascular disease(s) (Beta 0.21, $p=0.04$ ) and having an internal locus of control/Mastery (Beta of $-0.36, p=0.001$ ) were significant predictors of depression symptomatology. This model explained $38 \%$ of the variance. In women with Type 2 diabetes, statistically significant predictors of depression were: receiving instrumental support (Beta 0.28, $p=0.02$ ) and Mastery (Beta -0.25 , $p=0.013$ ), explaining $19 \%$ of the variance.

\section{Discussion}

The main aim of the study was to investigate the prevalence of pervasive depression in a community-based sample of people with Type 2 diabetes and to compare this rate with people without a chronic condition. The prevalence of Type 2 diabetes was $7.0 \%$ in this community-based sample of older adults, which resembles the prevalence of Type 2 diabetes found in other community-based epidemiological studies [21, 22, 23]. Our results show that one in every five patients with 
F. Pouwer et al.: Rates and risks for co-morbid depression in patients with Type 2 diabetes mellitus

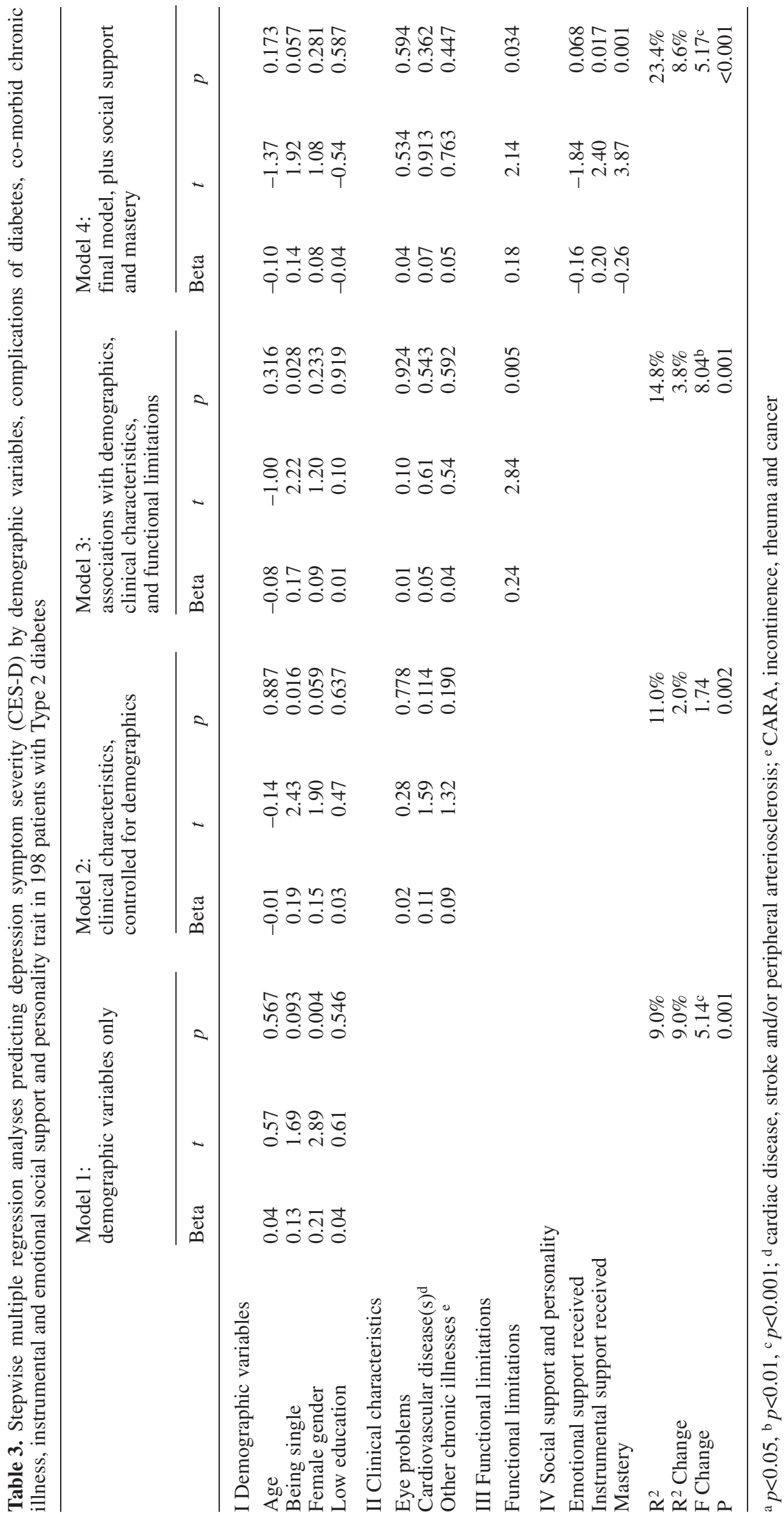


Type 2 diabetes and co-morbid disease suffered from pervasive depression. In contrast, patients with Type 2 diabetes without co-morbid disease appeared not to have a higher mean CES-D-depression scores compared with healthy subjects. Likewise, compared to healthy subjects, the odds for pervasive depression are particularly increased in subjects who have Type 2 diabetes and other co-morbid diseases, but not in those with Type 2 diabetes only. The presence of comorbid disease seemed to at least double the risk for pervasive depression in those with Type 2 diabetes. The results of our population-based study therefore confirm the conclusions of a recent review [1]. Furthermore, we found that more than $75 \%$ of the patients with Type 2 diabetes suffered from co-morbid chronic conditions, in addition to diabetes. Hence the majority of the patients with Type 2 diabetes have an increased risk for depression. Having multiple chronic conditions in addition to diabetes can be regarded as burdensome for many patients, as these conditions are associated with an increased number of functional limitations. Subsequently, functional limitations can negatively affect the patients' quality of life and thus contribute to the development of depression.

In the linear regression analyses, depression symptomatology was found to be positively associated with an external locus of control, having co-morbid disease, having functional limitations, having no partner and being female. This finding is in line with previous studies of several authors and suggest that many general life stresses, in addition to diabetes-related distress, can challenge the patients' adaptive capacities and can lead to a negative mood $[6,9,10,11,24,25]$.

There are several limitations that need to be mentioned. First, our measure of Type 2 diabetes was based on the self-report of the subjects. Within the framework of LASA, the accuracy of the patients' self-reports of several chronic diseases, as compared with general practitioners information was studied and the percentages of underreporters (19.9\%) and overreporters $(0.6 \%)$ was found to be the lowest for diabetes [26]. In sum, the overall concordance between patients and general practitioners was $97.8 \%$ for diabetes. Nevertheless, we might have missed a number of patients with Type 2 diabetes in an early stage of the disease, as many of these patients are not aware of the fact that they have diabetes. If this is the case, our prevalence rates of depression might be overestimated, as the prevalence of depression was found to be lower in people with newly diagnosed diabetes [1]. On the other hand, we might have also missed patients with Type 2 diabetes with severe diabetic complications, who were too ill to participate.

In sum, our results suggest that in particular complicated Type 2 diabetes is positively associated with depression. One in every five patients with Type 2 diabetes and a co-morbid chronic disease appeared to suffer from pervasive depression. With these findings and the results of other research groups, we can assume that depression is a common, serious and disabling complication of Type 2 diabetes in both men and women. Depression can be treated effectively in patients with diabetes [27, 28], but there is evidence to suggest that depression is under-recognized (and consequently under-treated) in this group of patients [29]. The use of a depression questionnaire to screen for depression can improve these detection rates [30, 31]. However, prospective studies are needed to test the hypothesis that screening for depression in patients with diabetes has beneficial effects on the outcomes of diabetes care.

Acknowledgements. Dr. Frans Pouwer is supported by a grant from The Netherlands Organisation for Scientific Research (NWO) grant number 940-34-007.

\section{References}

1. Anderson RJ, Freedland KE, Clouse RE, Lustman PJ (2001) The prevalence of comorbid depression in adults with diabetes: a meta-analysis. Diabetes Care 24:1069-1078

2. Jacobson AM, Groot M de, Samson JA (1997) The effects of psychiatric disorders and symptoms on quality of life in patients with type I and type II diabetes mellitus. Qual Life Res 6:11-20

3. Lloyd CE, Dyer PH, Barnett AH (2000) Prevalence of symptoms of depression and anxiety in a diabetes clinic population. Diabet Med 17:198-202

4. Pouwer F, Snoek FJ (2001) Association between symptoms of depression and glycaemic control may be unstable across gender. Diabet Med 18:595-598

5. Lustman PJ, Anderson RJ, Freedland KE et al. (2000) Depression and poor glycemic control: a meta-analytic review of the literature. Diabetes Care 23:934-942

6. Egede LE, Zheng D, Simpson K (2002) Comorbid depression is associated with increased health care use and expenditures in individuals with diabetes. Diabetes Care 25:464470

7. Talbot F, Nouwen A (2000) A review of the relationship between depression and diabetes in adults: is there a link? Diabetes Care 23:1556-1562

8. Groot M de, Anderson R, Freedland KE, Clouse RE, Lustman PJ (2001) Association of depression and diabetes complications: a meta-analysis. Psychosom Med 63:619630

9. Black SA, Goodwin JS, Markides KS (1998) The association between chronic diseases and depressive symptomatology in older Mexican Americans. J Gerontol A Biol Sci Med Sci 53:M188-94

10. Black SA (1999) Increased health burden associated with comorbid depression in older diabetic Mexican Americans. Results from the Hispanic Established Population for the Epidemiologic Study of the Elderly survey. Diabetes Care 22:56-64

11. Fisher L, Chesla CA, Mullan JT, Skaff MM, Kanter RA (2001) Contributors to depression in Latino and EuropeanAmerican patients with type 2 diabetes. Diabetes Care 24:1751-1757

12. White NE, Richter JM, Fry C (1992) Coping, social support, and adaptation to chronic illness. West J Nurs Res 14:211-224 
13. Macrodimitris SD, Endler NS (2001) Coping, control, and adjustment in Type 2 diabetes. Health Psychol 20:208216

14. Deeg DJH, Knipscheer CPM, Van Tilburg W (1993) Autonomy and well-being in the aging population: concepts and design of the Longitudinal Aging Study Amsterdam. NIG-Trendstudies no 7. Netherlands Institute of Gerontology, Bunnik, The Netherlands

15. Beekman AT, Deeg DJ, Tilburg T van, Smit JH, Hooijer Ch, Van Tilburg T (1995) Major and minor depression in later life: a study of prevalence and risk factors. J Affect Disord 36:65-75

16. Van Sonsbeek JLA (1988) Methodological and substantial aspects of the OECD indicator of chronic functional limitations. Maandbericht Gezondheid (CBS) 88:4-17

17. Radloff LS (1977) The CES-D scale: a self-report depression scale for research in the general population. Appl Psychol Meas 1:385-401

18. Beekman AT, Deeg DJ, Van Limbeek J, Braam AW, De Vries MZ, Van Tilburg W (1997) Criterion validity of the Center for Epidemiologic Studies Depression scale (CES-D): results from a community-based sample of older subjects in The Netherlands. Psychol Med 27:231-235

19. Pearlin LI, Schooler C (1978) The structure of coping. J Health Soc Behav 19:2-21

20. Tilburg $\mathrm{T}$ van (1998) Losing and gaining in old age: changes in personal network size and social support in a four-year longitudinal study. J Gerontol 53:S313-323

21. Mooy JM, Grootenhuis PA, Vries H de et al. (1995) Prevalence and determinants of glucose intolerance in a Dutch caucasian population. The Hoorn Study. Diabetes Care 18:1270-1273

22. Heine RJ, Nijpels G, Mooy JM (1996) New data on the rate of progression of impaired glucose tolerance to NIDDM and predicting factors. Diabet Med 13 (3 Suppl 2): S12-14
23. Bourdel-Marchasson I, Dubroca B, Manciet G et al. (1997) Prevalence of diabetes and effect on quality of life in older French living in the community: the PAQUID Epidemiological Survey. J Am Geriatr Soc 45:295-301

24. Palinkas LA, Wingard DL, Barrett-Connor E (1990) Chronic illness and depressive symptoms in the elderly: a population-based study. J Clin Epidemiol 43:1131-1141

25. Palinkas LA, Barrett-Connor E, Wingard DL (1991) Type 2 diabetes and depressive symptoms in older adults: a population-based study. Diabet Med 8:532-539

26. Kriegsman DM, Penninx BW, Eijk JT van, Boeke AJ, Deeg DJ (1996) Self-reports and general practitioner information on the presence of chronic diseases in community dwelling elderly. A study on the accuracy of patients' selfreports and on determinants of inaccuracy. J Clin Epidemiol 49:1407-1417

27. Lustman PJ, Freedland KE, Griffith LS, Clouse RE (2000) Fluoxetine for depression in diabetes: a randomized double-blind placebo-controlled trial. Diabetes Care 23:618623

28. Lustman PJ, Griffith LS, Freedland KE, Kissel SS, Clouse RE (1998) Cognitive behavior therapy for depression in type 2 diabetes mellitus. A randomized, controlled trial. Ann Intern Med 129:613-621

29. Lustman PJ, Harper GW (1987) Nonpsychiatric physicians' identification and treatment of depression in patients with diabetes. Compr Psychiatry 28:22-27

30. Lustman PJ, Clouse RE, Griffith LS, Carney RM, Freedland KE (1997) Screening for depression in diabetes using the Beck Depression Inventory. Psychosom Med 59:24-31

31. Pouwer F, Snoek FJ, Ploeg HM van der, Ader HJ, Heine RJ (2001) Monitoring of psychological well-being in outpatients with diabetes: effects on mood, $\operatorname{HbA}(1 c)$, and the patient's evaluation of the quality of diabetes care: a randomized controlled trial. Diabetes Care 24:1929-1935 